Lung cancer

\section{Screening for lung cancer: yet another problem}

\section{Stephen G Spiro}

\section{Implementation of a mass screening programme for lung cancer}

L ung cancers present late, allowing most sufferers little chance of curative - treatment. Presenting symptoms are usually non-specific and give the primary-care physicians little to look out for to separate the presentation of lung cancer, which is the most serious of diseases, from the common cold, a respiratory tract infection, fibromyalgia or any other self-limiting condition of little long-term consequence that he or she sees in daily abundance. ${ }^{1}$ It has been known for years however, that the most favourable presentation for lung cancer is the chance discovery on a routine chest radiograph, and this was one of the drivers for the chest radiograph to be used as a screening tool for lung cancer in the 1950s and 1960s. However, although chest radigraph screening studies, where usually individuals are randomised to an $x$ ray annual screen or simple follow-up, did disclose more cancers in the active study arm, their mortality was not improved. $^{2-4}$ These studies have been criticised for several reasons, including inadequate number of entrants, noncompliance in the follow-up only arm and claims that, maybe after all, there was a potential advantage in mortality for the screen-discovered cancers. Nevertheless, the issue was dropped until the development of low-dose multi-slice computed tomography scanning made computed tomography suitable for studying large numbers of people with only a small radiation exposure. Over the past 10 years, there have been several hypothesis-generating studies on populations with differing degrees of risk for developing lung cancer who have undergone prevalence and incidence computed tomography screening in non-randomised studies. These studies have been carried out mainly in Japan and the United States, and have incorporated healthy volunteers of middle or late age (45 years or more), of sufficient health status to be likely to live for at least another 5 years (so as to be able to undergo pulmonary resection, survive and be likely evaluable for cure), and to be a current or ex-smoker (usually of at least 20 pack years), and in some, to have increased airflow obstruction (a measure associated with an increased risk for lung cancer)..$^{5-9}$ All these studies found an up to fourfold higher prevalence of lung cancers than reported for the chest radiograph. The prevalence depended on the age of the population, the number of pack years smoked and the presence of airways obstruction, with an overall prevalence of between $1.3 \%$ and $2.8 \%$. Furthermore, the majority of prevalence cancers were stage IA, although, in the much smaller number of incidence cancers found at annual screening, this favourable stage shift did not occur as frequently. However, even this haul of asymptomatic cancers of apparently favourable stage ignores important biases. Firstly, the fact that more aggressive tumours will emerge with presenting symptoms between annual screens (length-time bias). Also, screening may overdiagnose lung cancer and, in particular, those more indolent tumours that may not cause death of an individual (overdiagnosis bias); and screening would also need to show a positive effect on mortality to ensure that better 5-year survival owing to discovery at an early, more favourable stage is not just due to lead-time bias.

There could be other criticisms of these computed tomography based studies that may be relevant to other countries where lung cancer remains common. These studies were carried out in countries where adenocarcinoma remains the commonest cell type and is usually a peripheral tumour, making detection by computer tomography more likely. Very few centrally located tumours are identified by this screening method, and in Europe, where squamous cancers still predominate, they are less easily identified by computed tomography as they tend to arise from the central airways. Similarly, few small cell cancers are found by screening owing to their aggressive nature and the probability that they will present with symptoms and will only rarely be a chance discovery. Screening with computed tomography, therefore, will identify only a proportion of lung cancers depending, to some extent, on their site of origin (peripheral or central) and on their biological growth rates. Another problem is the presence of benign non-calcified nodules which have the potential to be confused with a tumour, also defined as a non-calcified nodule. The increasing experience of radiologists reporting screening scans and an algorithmic approach to these nodules has led to fewer unnecessary operations being carried out in centres which have focussed on screening, but there remains an important learning curve for radiologists, physicians and surgeons involved in these studies. The incidence of benign nodules will vary on where in the world the subject lives, with the incidence of nodules on computed tomography varying from up to $70 \%$ in the mid-west United States to about 10 $20 \%$ in Japan and some European areas..$^{8-10}$ Then, once a nodule is discovered, some advocate conventional (higher radiation dose) computed tomography to delineate the lesion more clearly, and often a regular follow-up computed tomography every 36 months for up to a year or more, with all the consequent exposure to radiation as well as the worry and uncertainty for the individuals concerned.

Ultimately, if this screening tool has to come into use, it has to prove its capability of improving the disease-specific mortality from lung cancer. To do this, there have to be randomised controlled trials, although others have argued that this would be unnecessary, based on "diagnostic-prognostic trials". These trials studied two component issues of screening, the diagnostic regimen and its prognostic implications. Evaluation of the screening regimen, the diagnostic component, it is argued, requires no control group, as the information about the performance of the regimen can be obtained from those receiving it. ${ }^{11}$ Nevertheless, there are several randomised controlled trials in progress, and some have already closed to recruitment. Perhaps the biggest and the most expensive randomised controlled trial is the National Lung Cancer Screening Trial in the United States, in which 50000 individuals aged over 45 years, with a smoking history of 20 pack years were randomised to a prevalence computed tomography and three annual incidence screens versus chest radiographs at identical timings. This study is likely to cost at least \$200 million, and, as with the other studies, will need a follow-up period after closure of 5 years, making results unavailable for 9-10 years. The other ongoing studies are mainly in Europe, and are recruiting much smaller numbers and vary as to whether individuals in the 
control arm have an annual $x$ ray or nothing other than simple follow-up. The contamination rate for the control arm (ie, having a sneak chest radiograph or even a privately paid computed tomography) is not known, although this reached in excess of $50 \%$ in the chest radiograph versus nothing randomised controlled trial led by the Mayo Clinic in the 1950s.

Into this area of uncertainty comes a most interesting study with perhaps an intuitively obvious result from Silvestri et al in this month's issue of Thorax (see page 126)..$^{12}$ They carried out a telephone questionnaire on a nationwide sample of Americans aged $\geqslant 40$ years, identified by a random dialling telephone system. From 21000 households contacted, 2001 individuals were prepared to answer structured questions from a trained interviewer. What transpired was that smokers in the survey were significantly more likely than non-smokers to be male, nonwhite, less well educated and to report poorer health status or history of cancer, and also (in the US system) to be less able to identify a usual source of healthcare. When contrasting the attitude to screening for risk of disease, the current smokers were less likely than the nonsmokers to believe that early detection would result in a good chance of survival. The smokers were also less likely to consider undergoing a computed tomography for screening for lung cancer, and fewer current smokers believed that the risk of lung cancer or the accuracy of the test was an important factor in deciding whether to enter a screening programme. Even worse, only half of the current smokers would have chosen to opt for surgical removal of a screen-diagnosed cancer.

The authors of the paper are correct to conclude that there remain substantial obstacles to the implementation of a mass screening programme for lung cancer. Many of the problems have been outlined above, without mentioning the huge effect the organisation of such a programme would have on resource, equipment, radiographers, radiologists, surgeons and, not least, the funders. There is even a debate ongoing on the best design for such a programme. Also, with the rapidly improving technology around sophisticated pulmonary imaging, it is hard to see how a 5-year study can be set up without the means to incorporate newer imaging computer programs and other refinements as the study progresses. What about the role of positron emission tomography which is regarded as more sensitive and specific than computed tomography alone in determining the potential malignant nature of a lesion? On top of this are data suggesting that those who we would wish to be the primary targets to screening may be the most indifferent to its benefits and unwilling to participate. It would be of little use if the educated, affluent and interested were the only recepients of this expensive tool. However, despite all the negative arguments, the value of screening remains unproved and therefore continues as a very important issue in the management of lung cancer, and should be properly supported and researched until its value becomes clear.
Thorax 2007:62:105-106.

doi: $10.1136 /$ thx.2006.061309

Correspondence to: Professor S G Spiro, Department of Respiratory Medicine, University College Hospital, Grafton Way, London WCIE 6AU, UK; stephen.spiro@uclh.nhs.uk

\section{REFERENCES}

1 Hamilton W, Peters TJ, Round A, et al. What are the clinical features of lung cancer before the diagnosis is made? A population based case-controlled study. Thorax 2005;60: 1059-65.

2 Fontana RS, Sanderson DR, Woolner LB, et al. Lung cancer screening. The Mayo Program. J Occup Med 1986;28:746-50.

3 Marcus P, Bergstralh E, Fagerstrom R, et al. Lung cancer mortality in Mayo Lung Project: impact of extended follow-up. I Natl Cancer Inst 2000;92:1308-16.

4 Bach P, Kelly $M$, Tate $R$, et al. Screening for lung cancer. A review of the literature. Chest 2003; 123:72S-82S.

5 Henschke C, McCauley D, Yankelevitz D, et al. Early lung cancer detection project: overall design and findings from baseline screening. Lancet 1999;354:99-105.

6 Sone S, Takashima S, Li F, et al. Mass screening for lung cancer with mobile spiral computed tomography scan. Lancet 1998;351:1242-5.

7 Sobue T, Moriyama N, Kaneko M, et al. Screening for lung cancer with low-dose helical computed tomography: anti-lung cancer association project. J Clin Oncol 2002;20:91 1-20.

8 Swensen S, Jett J, Sloan J, et al. Screening for lung cancer with low-dose spiral computed tomography. Am J Respir Crit Care Med 2002;165:508-13.

9 MacRedmond R, Logan PM, Lee M, et al. Screening for lung cancer using low dose CT scanning. Thorax 2004:59:237-41.

10 Swensen S, Jeft J, Hartman T, et al. Lung cancer xcreening with CT: Mayo Clinic experience. Radiology 2003:226:756-61.

11 Henschke Cl, Reeves AP, Shaham D, et al. CT screening for lung cancer. In:Textbook of prevention and detection of early lung cancer.Taylor and Francis, 2005: 187-204

12 Silvestri GA, Nietert PJ, Zoller J, et al. Attitudes towards screening for lung cancer among smokers and their non-smoking counterparts. Thorax 2006;62: 126-30.

\section{Further evidence that the wealthier are healthier: negative life events and asthma-specific quality of life}

\section{R J Wright}

\section{Association between psychological stress and asthma expression and morbidity}

n this issue of Thorax, Archea et al ${ }^{1}$ (see $p$ 139) add to the growing number of studies linking psychological stress to asthma expression and morbidity. To date, studies have reported an association between various measures of life stress and both the onset of asthma or precedent phenotypes ${ }^{23}$ and exacerbations of established disease. ${ }^{4-6} \quad$ Hypothesised mechanisms underlying the association between stress and asthma expression implicate reciprocal relationships between neural, hormonal and immunological pathways that have been extensively reviewed previously. ${ }^{78}$ Evidence demonstrating that psychological stress influences the expression of inflammatory cytokine patterns in patients with asthma or those at risk of developing the disease supports these theories. ${ }^{9}{ }^{10}$ Others have shown that physiological changes, including neuroimmune and genetic processes, may lead to differential responses to therapeutic interventions for asthma and atopic disorders. ${ }^{11}{ }^{12}$

The current work by Archea et al underscores that psychological factors may also be more indirect, albeit equally important, determinants of asthma 